\title{
Point Group Interpretation of Galois Symmetry of Bethe Ansatz Solutions of Magnetic Pentagonal Ring
}

\author{
B. LULEK ${ }^{a}$, T. LULEK ${ }^{b, *}$, M. EABUZ ${ }^{c}$ AND J. MILEWSKI ${ }^{d}$ \\ ${ }^{a}$ East European State Higher School, T. Terleckiego 6, 37-700 Przemyśl, Poland \\ ${ }^{b}$ Faculty of Physics, Adam Mickiewicz University, Umultowska 85, 61-614 Poznań, Poland \\ ${ }^{c}$ Department of Theoretical Physics, Faculty of Mathematics and Natural Sciences, University of Rzeszow, \\ S. Pigonia 1, 35-310 Rzeszów, Poland \\ ${ }^{d}$ Institute of Mathematics, Poznan University of Technology, Piotrowo 3A, 60-965 Poznań, Poland

\begin{abstract}
Exact solutions of the eigenproblem of the magnetic pentagonal ring exhibit the arithmetic symmetry expressed in terms of a Galois group of a finite extension of the prime field $\mathbb{Q}$ of rationals. We propose here a geometric interpretation of this symmetry in the interior of the Brillouin zone, in terms of point groups. Explicitly, it is a subgroup of the direct product $C_{4} \times D_{4}$. We present also the appropriate irreducible representations of the group.
\end{abstract}

DOI: 10.12693/APhysPolA.127.336

PACS: 75.10.Jm, 03.65.Fd, 03.67.Lx, 02.10.Ud, 02.10.De

\section{Introduction}

Bethe Ansatz [1-4] provides unique exact solution of the eigenproblem of the isotropic Heisenberg Hamiltonian for a magnetic ring of $N$ nodes with the spin $1 / 2$. Recently, it has been demonstrated that this solution exhibits a Galois symmetry, stemming from the fact that the eigenproblem in the initial basis of magnetic configurations is expressible in integers, and thus the solutions require only a finite extension of the prime field $\mathbb{Q}$ of rationals [5-8]. The Galois group of this field extension has proven to be a useful tool in discovering several algebraic symmetries between exact eigenvalues and eigenstates. Details of this tool are expressed, however, in a somehow hermetic terms of algebraic Galois theory. We intend to interpret them in terms of quantum mechanical notions and calculations. The main aim of the present paper is interpretation of the Galois group for the magnetic pentagon $(N=5)[5,6]$ in terms of some symmetries of point groups.

\section{The eigenproblem of the pentagon and Bethe Ansatz}

A detailed description of the diagonalisation procedure for the magnetic pentagon has been given in [5]. Here we focus our attention on the two-magnon sector $(r=2$ spin deviations from the ferromagnetic saturation), in the interior $B_{\text {int }}=\{k= \pm 1, \pm 2\}$ of the Brillouin zone for the pentagon, where $k$ is the quasimomentum, the exact quantum number responsible for the translational symmetry of the pentagon, given by the cyclic group $C_{5}$. The $10 \times 10$ block Heisenberg Hamiltonian for the sector $r=2$, consisting of integers ( 2 or 4 on the main diagonal, and

${ }^{*}$ corresponding author; e-mail: tadlulek@amu.edu.pl
1 or 0 outside), decomposes into two-dimensional blocks of the form given in detail in [5]. In principle, an arbitrary quantum mechanical eigenproblem in the finitedimensional Hilbert space assumes the number field $\mathbb{C}$ of complex numbers, as underlying number field. The field $\mathbb{C}$ is algebraically complete, i.e., any polynomial with coefficients in $\mathbb{C}$ has all its roots in $\mathbb{C}$. However, in many cases, including our case of pentagon, this field is redundant, in spite of the following facts:

(a) the original $10 \times 10$ problem in the basis of magnetic configurations requires only the prime field $\mathbb{Q} \subset \mathbb{C}$ (since all matrix elements are integers),

(b) the effective four $2 \times 2$ eigenproblems for the interior $B_{\text {int }}$ require the cyclotomic extension $\mathbb{Q}(\omega)$ of the prime field $\mathbb{Q}$,

(c) solutions of the eigenproblem are also expressible in $\mathbb{Q}(\omega)$. Due to this fact, $\mathbb{Q}(\omega)$ can be referred to as to the Heisenberg field of magnetic pentagon [6, 7].

The solution obtained from $2 \times 2$ eigenproblems do not display explicitly the string structure of the Bethe Ansatz solutions. This structure can be derived by solution of the so called inverse Bethe Ansatz [5-9]:

$$
\begin{aligned}
& a_{k} b_{k}=\omega^{-k}, \\
& a_{k}+a_{k}^{-1}+b_{k}+b_{k}^{-1}=E_{k}+4,
\end{aligned}
$$

where $a_{k}$ and $b_{k}$ are unknown portions of phase related to spectral paramters $(\lambda, \mu)$ and pseudomomenta $p_{1}, p_{2}$, $\omega=\exp (2 \pi \mathrm{i} / 5)$, and $E_{k}=-4+(-1)^{k} \sqrt{5}, k \in B_{\text {int }}$, stands for eigenenergies of the problem being discussed. Equation (1) has a simple physical meaning of conservation of quasimomentum and energy. According to [6], Bethe parameters $a_{k}, b_{k}$ can be obtained in the form

$$
\left(a_{k}, b_{k}\right)=\frac{(-1)^{k} \sqrt{5} \pm \gamma_{\epsilon(k)}}{2\left(1+\omega^{k}\right)}, \quad k \in B_{\text {int }},
$$

where $\epsilon: \mathbb{Z}_{5}^{\times} \rightarrow C_{2}$ is the homomorphism given by $\epsilon(2)=-1$, and $\gamma_{1}=\mathrm{i} \sqrt{1+2 \sqrt{5}}, \gamma_{-1}=\sqrt{-1+2 \sqrt{5}}$, so that $\gamma_{1}$ and $\gamma_{-1}$ corresponds to scattered $(k= \pm 1)$ 
and bound ( $k= \pm 2$ ) states of pentagon, denoted by

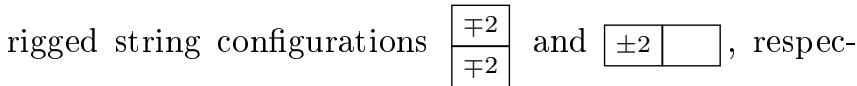
tively [10-12].

The Bethe Ansatz solution of the eigenproblem is expressible within the extension of the cyclotomic field $\mathbb{Q}(\omega)$ by $\gamma_{1}$ and $\gamma_{-1}$. This extension can be denoted by $\mathcal{B}=\mathbb{Q}\left(\omega, \gamma_{1}, \gamma_{-1}\right)$, and referred to as the Bethe field.

\section{Galois symmetries}

The Bethe field $\mathcal{B}$ is a 16 -dimensional linear space over $\mathbb{Q}$, with the basis (cf. [6]):

$$
\left[k ; l_{1}, l_{2}\right]:=\gamma_{1}^{l_{1}} \gamma_{-1}^{l_{-1}} \omega^{k}, \quad l_{1}, l_{2} \in \mathbb{Z}_{2}, k \in \mathbb{Z}_{5}^{\times} .
$$

Within this context, the interior $B_{\text {int }}$ of the Brillouin zone for pentagon can be identified with the multiplicative group $\mathbb{Z}_{5}^{\times}$of the finite number field $\mathbb{Z}_{5}$, and the ranges for $l_{1}$ and $l_{-1}$ with $\mathbb{Z}_{2}$. The Galois group of the Bethe field $\mathcal{B}$, i.e. the group $G=\operatorname{Aut}(\mathcal{B} / \mathbb{Q})$ of all automorphisms of $\mathcal{B}$ acts on the basis (3) as

$$
\left(l ; \eta_{1}, \eta_{-1}\right)\left[k ; l_{1}, l_{-1}\right]=\eta_{1}^{l_{1}} \eta_{-1}^{l_{-1}}\left[l k ; l_{\epsilon(k)}, l_{-\epsilon(k)}\right],
$$
such that

$$
G=\left\{\left(l ; \eta_{1}, \eta_{-1}\right) \mid \eta_{1}, \eta_{-1} \in C_{2}, l \in \mathbb{Z}_{5}^{\times}\right\},
$$

where $C_{2} \equiv\{1,-1\}$ can be interpreted as the cyclic group $C_{2}$ with the multiplicative group composition (to be distinguished from the additive group $\left.\left(\mathbb{Z}_{2},+\right)\right)$. Equation (4) yields the multiplication law

$$
\left(l^{\prime} ; \eta_{1}^{\prime}, \eta_{-1}^{\prime}\right)\left(l ; \eta_{1}, \eta_{-1}\right)=\left(l^{\prime} l ; \eta_{\epsilon(l)}^{\prime} \eta_{1}, \eta_{-\epsilon(l)}^{\prime} \eta_{-1}\right) .
$$

Equation (6) shows that the group $G$ is a semidirect product

$$
G=\mathbb{Z}_{5}^{\times} \times{ }_{\psi}\left(C_{2} \times C_{2}\right),
$$
with $\psi: \mathbb{Z}_{5}^{\times} \rightarrow \operatorname{Aut}\left(C_{2} \times C_{2}\right)$ given by $\psi(l)\left(\eta_{1}, \eta_{-1}\right)=$ $\left(\eta_{\epsilon(l)}, \eta_{-\epsilon(l)}\right)$, being the action of the active group $\mathbb{Z}_{5}^{\times}$on the passive group $C_{2} \times C_{2}$, which permutes only the order of elements in the semidirect product $C_{2} \times C_{2}$, so that the group (7) is the wreath product of the group $C_{2}$ and $C_{4}$. It determines the action of the Galois group $G$ in the Bethe field $\mathcal{B}$, in the basis (3) adapted to exact Bethe Ansatz eigenstates. It thus allows one to generate all eigenstates within the interior $B_{\text {int }}$ of the Brillouin zone from a single one. We describe the group $G$ as the Bethe-Galois group for the interior of the Brillouin zone for the Heisenberg pentagon.

\section{Point group extensions}

The chain of subfields $\mathbb{Q} \subset \mathbb{Q}(\omega) \subset \mathcal{B}$ implies that the Bethe-Galois group $G$ is the extension of the (Abelian) group

$$
D_{2}=\operatorname{Aut}(\mathcal{B} / \mathbb{Q}(\omega))=\left\{\left(l_{1}, l_{-1}\right) \mid l_{1}, l_{-1} \in \mathbb{Z}_{2}\right\},
$$

isomorphic to the dihedral point group $D_{2}$, by the group

$$
C_{4}=\operatorname{Aut}(\mathbb{Q}(\omega) / \mathbb{Q})=\mathbb{Z}_{5}^{\times} \cong B_{\text {int }},
$$

isomorphic to the cyclic point group $C_{4}$, in accordance with the short exact sequence of groups and homomorphisms

$$
1 \rightarrow D_{2} \rightarrow G \rightarrow C_{4} \rightarrow 1
$$

Roughly speaking, the passive group changes at most only the ring of numbers $\gamma_{1}$ and $\gamma_{-1}: \gamma_{1} \longmapsto \pm \gamma_{1}$, $\gamma_{-1} \longmapsto \pm \gamma_{-1}$, whereas the active group $C_{4}$ permutes quasimomenta in the interior of the Brillouin zone. The combined action of these two groups, described precisely by Eq. (6), admits transformations of bound and scattered two-magnon states, accompanied by a gauge by \pm 1 .

\section{Geometric interpetation}

The Bethe-Galois group $G$, presented in previous sections as the extension of $D_{2}$ by $C_{4}$ with the operator action $\psi$, can be also seen as a subgroup of the index 2 , embedded into the direct product $D_{4} \times C_{4}$ of standard point groups. Within this setting, $G$ acts on two squares, presented in Fig. 1. Vertices of the first square represent the regular orbit of the defining action of $C_{4}$ as the Galois group of the cyclotomic field along Eq. (9), whereas those of the second - a transitive representation of the point group $D_{4}$, having $D_{2}$ of Eq. (8) as its subgroup.
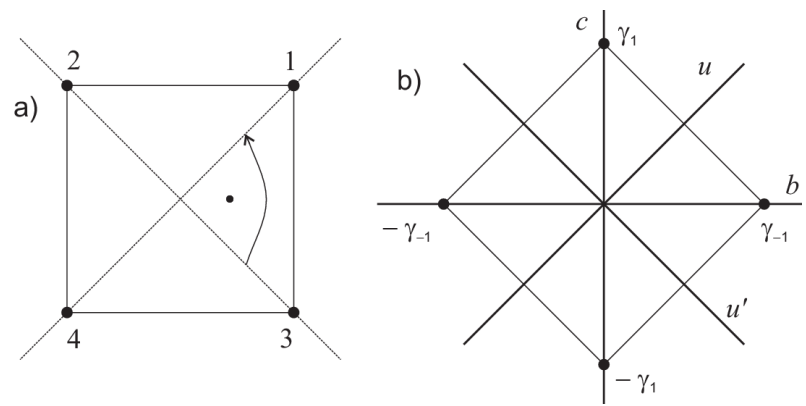

Fig. 1. The action of Galois group on two squares: (a) passive group, (b) active group.

On the first square there acts the group $C_{4}=\left\langle c_{4}\right\rangle$, which is generated by the element $c_{4}$, corresponding to the rotation by the $\pi / 2$ angle. It is compatible with a multiplication of an index of vertices by $2 \bmod 5$. On the second square there acts the group $D_{4}$, which, except of rotations by a multiple of the right angle, contains also reflections versus $b, c, u$ and $u^{\prime}$ axes. For both groups, $C_{4}$ and $D_{4}$, one can distinguish adequate subgroups of the rank 2: $C_{4}^{2}:=\left\langle c_{4}{ }^{2}\right\rangle$ in $C_{4}$, and $D_{2}:=\langle b, c\rangle$ in $D_{4}$. The Bethe-Galois group is isomorphic with the group $G$ :

$$
G=\left(C_{4}^{2} \times D_{2}\right) \cup\left(c_{4} C_{4}^{2} \times c_{4} D_{2}\right) .
$$

Now we see that groups described by Eqs. (5) and (11) are isomorphic. Indeed, the isomorphism is given by generators of these groups [6]:

$$
\tilde{a}=\left(c_{4}, u\right) \cong(2 ; 1,1), \quad \tilde{b}=(\mathrm{e}, b) \cong(1 ;-1,1) .
$$

The form (11) is convenient to make a split of the group $G$ into classes of conjugated elements. As all the classes from the group $C_{4}$ are single-element (like in every Abelian group), and taking into account classes in the group $D_{4}$ of the form $\{\mathrm{e}\},\left\{c_{4}, c_{4}^{-1}\right\},\left\{c_{4}^{2}\right\},\{a, b\},\left\{u, u^{\prime}\right\}$, 
one gets the split of Bethe-Galois group into 4 oneelement classes

$$
\{(\mathrm{e}, \mathrm{e})\}, \quad\left\{\left(\mathrm{e}, c_{4}{ }^{2}\right)\right\}, \quad\left\{\left(c_{4}{ }^{2}, \mathrm{e}\right)\right\}, \quad\left\{\left(c_{4}{ }^{2}, c_{4}{ }^{2}\right)\right\},
$$

and 6 two-element classes

$$
\begin{aligned}
& \left\{\left(c_{4}{ }^{l}, c_{4}\right),\left(c_{4}{ }^{l}, c_{4}{ }^{-1}\right)\right\} ; \quad l= \pm 1, \\
& \left\{\left(c_{4}{ }^{l}, b\right),\left(c_{4}{ }^{l}, c\right)\right\} ; \quad l=0,2, \\
& \left\{\left(c_{4}{ }^{l}, u\right),\left(c_{4}{ }^{l}, u^{\prime}\right)\right\} ; \quad l= \pm 1 .
\end{aligned}
$$

Furthermore, the formula (11) gives possibility of construction of irreducible representations of the group $G$ via representation of the group $C_{4}$ and $D_{4}$. Appropriate matrices of irreducible representations are of the form

$$
\begin{gathered}
\tau_{n, m}(\tilde{b})=(-1)^{n}, \tau_{n, m}(\tilde{a})=\mathrm{i}^{m}, \\
n \in \mathbb{Z}_{2}, m \in \mathbb{Z}_{4},
\end{gathered}
$$

for one-dimensional representations, and

$$
\begin{aligned}
& E_{0}(\tilde{b})=E_{1}(\tilde{b})=\left(\begin{array}{rr}
1 & 0 \\
0 & -1
\end{array}\right) \\
& E_{0}(\tilde{a})=\left(\begin{array}{ll}
0 & 1 \\
1 & 0
\end{array}\right), \quad E_{1}(\tilde{a})=\left(\begin{array}{rr}
0 & \mathrm{i} \\
\mathrm{i} & 0
\end{array}\right)
\end{aligned}
$$

for two-dimensional representations. One can distinguish eight one-dimensional representations, and two two-dimensional representations, which is concordant with the Burnside theorem $8 \cdot 1^{2}+2 \cdot 2^{2}=16$.

\section{Conclusions}

We have proposed an interpretation of the Galois symmetries of the Bethe Ansatz solutions for magnetic pentagonal ring inside its Brillouin zone, expressed by socalled Bethe group, in terms of point groups $C_{4}, D_{2}$, and $D_{4}$. Namely, the semidirect product of $D_{2}$ by $C_{4}$ can be embedded as a subgroup of index 2 in the direct product $C_{4} \times D_{4}$, which admits a geometric interpretation of actions of the relevant group on two deformed squares in the complex plane. It makes transparent the structure of conjugacy classes and irreducible representations of the Bethe group. In particular, bound and scattered two-magnon states are interchanged by the action of the Bethe group.

\section{Acknowledgments}

The research was partially supported by the grant: NCN 2013/09/B/ST1/04416.

\section{References}

[1] H. Bethe, Z. Phys. 71, 205 (1931) (in German); English translation in: D.C. Mattis, The Many-Body Problem, World Sci., Singapore 1993, p. 689.

[2] R.J. Baxter, J. Stat. Phys. 108, 1 (2002).

[3] W.J. Caspers, Spin Systems, World Sci., Singapore 1989.

[4] W.J. Caspers, B. Lulek, T. Lulek, M. Kuzma, A. Wal, Int. J. Mod. Pys. B 18, 1277 (2004).

[5] J. Milewski, G. Banaszak, T. Lulek, M. Labuz, Physica B 406, 520 (2011)

[6] G. Banaszak, B. Lulek, T. Lulek, J. Milewski, B. Szydło, Rep. Math. Phys. 71, 205 (2013).

[7] J. Milewski, G. Banaszak, T. Lulek, M. Labuz, R. Stagraczynski, OSID 19, 1250012 (2012).

[8] J. Milewski, B. Lulek, T. Lulek, M. Labuz, R. Stagraczynski, Physica B 434, 14 (2014).

[9] B. Lulek, T. Lulek, A. Wal, P. Jakubczyk, Physica B 337, 375 (2003).

[10] S.O. Warnaar, J. Stat. Phys. 82, 657 (1996).

[11] S. Dasmahapatra, O. Foda, Int. J. Mod. Phys. 38 , 1041 (1997).

[12] B. Lulek, T. Lulek, M. Labuz, R. Stagraczynski, Physica B 405, 2654 (2010). 